\title{
Assessing Genetic Diversity of Potato (Solanum tuberosum) Genotypes Grown in Tarai Region of Uttarakhand by Using Simple Sequence Repeat (SSR) Technique
}

\author{
Rajani*, Dhirendra Singh and A.S. Jeena \\ Department of Vegetable Science, G. B. Pant University of Agriculture and Technology, India \\ *Corresponding author
}

\begin{tabular}{|c|c|}
\hline & A B S T R A C T \\
\hline & \multirow{8}{*}{$\begin{array}{l}\text { This study aimed to evaluate the genetic diversity and identify potato cultivars by } \\
\text { SSR markers. The genomic DNA of } 50 \text { potato cultivars was amplified with } \\
\text { nineteen Simple Sequence Repeats (SSRs) primers. Out of nineteen primers only } \\
\text { fifteen primers were amplified and gave polymorphic bands. Therefore, fifteen } \\
\text { SSR primers were used in diversity analysis of } 50 \text { potato genotypes. Out of fifteen } \\
\text { primers, four primers were found to be monomorphic and rests were polymorphic. } \\
11 \text { SSR primers pairs which were amplified polymorphic band that generated } 34 \\
\text { polymorphic bands. Most of the primers detected more than one loci and observed } \\
\text { to be polymorphic thus able to differentiate different genotypes. The dendrograms } \\
\text { generated by cluster analysis distinguished the cultivars genetically. The PIC } \\
\text { values demonstrated the high information content of the primers used and } 50 \\
\text { potato varieties were identified based on SSR primer pairs. Thus, by means SSR } \\
\text { markers the genetic diversity was assessed and the } 50 \text { potato genotypes analyzed } \\
\text { in this study were identified. }\end{array}$} \\
\hline Keywords & \\
\hline Solanum & \\
\hline tuberosum, & \\
\hline $\begin{array}{l}\text { Micro-satellites, } \\
\text { Genetic diversity. }\end{array}$ & \\
\hline Article Info & \\
\hline $\begin{array}{l}\text { Accepted: } \\
\text { 29 June } 2017 \\
\text { Available Online: } \\
\text { 10 July } 2017\end{array}$ & \\
\hline & \\
\hline
\end{tabular}

\section{Introduction}

Genetic diversity is a prerequisite for an effective plant breeding programme. It is a useful and essential tool for parent's choice in hybridization to develop high yield potential cultivars and to meet the diversified goals of plant breeding (Gaur et al., 1978, Hayder et al., 2007 and Shekhawat, 2001).

The cultivated potato have narrow genetic base due to limited introduction of germplasm from their natural range in South America (Rauf et al., 2010). Most of the potato cultivars are autotetraploid $(2 \mathrm{n}=4 \mathrm{x}=48)$, highly heterozygous and out breeding species, which suffer from inbreeding depression. Heterosis in potato perpetuates because of its vegetative propagated nature. Therefore, selection of suitable parents for hybridization is crucial and evaluation of germplasm is essential to the development of new potato cultivars. The advantage of detecting polymorphism simply and quickly, but it lacks reproducibility (Chakrabarti et al., 2001), while SSR markers provide high reproducibility and genetic informativeness (Braun and Wenzel, 2005). In the present investigation, the purpose of the study was to evaluate the genetic diversity in 50 potato 
(Solanum tuberosum L.) genotypes that are being made available to potato growers and to develop a molecular profile using SSR markers.

\section{Materials and Methods}

The present investigation was conducted at Vegetable Research Centre (VRC) and Molecular diversity analysis was carried out in the Pantnagar Centre for Plant Genetic Resources (PCPGR) of Govind Ballabh Pant University of Agriculture \& Technology, Pantnagar, Udham Singh Nagar, Uttarakhand, India during 2014-15.

\section{Genomic DMA isolation protocol}

\section{Modified CTAB (Cetyl Trimethyl Ammonium Bromide) method}

Using electronic balance $1.0 \mathrm{~g}$ of alcohol sterilized leaf material was weighed for DNA extraction.

A minute quantity of PVP (Poly vinyl pyrrolidine) was added to combat the elect of phenolic compounds

Pre-weighed leaf material was ground in liquid nitrogen to tine powder using prechilled pestle and mortar

The powder was transferred to $15 \mathrm{ml}$ polypropylene tubes containing $5 \mathrm{~mL}$, of prewarmed extraction buffer. Spatula was used to dispense the material completely.

Samples were incubated at $60^{\circ} \mathrm{C}$ for $30 \mathrm{~min}$ with occasional mixing by gentle swirling.

$3 \mathrm{ml}$ (double amount of sample) chloroform: iso-amyl alcohol (241) was added and mixed by inversion to emulsify.

Centrifugation was done at $15,000 \mathrm{rpm}$ at room temperature for $10 \mathrm{~min}$.
Aqueous phase was removed with a wide bore pipette and transferred to a clean tube and added with $2 / 3$ volume of isopropanol, mixed by quick gentle inversion and the tubes were kept overnight at $-20^{\circ} \mathrm{C}$.

The samples were centrifuged for $10 \mathrm{~mm}$ at $5.000 \mathrm{rpm} .4^{\circ} \mathrm{C}$.

The supernatant was poured without disturbing the DNA pellet at the bottom of the tubes.

The DNA pellet was washed with $70 \%$ ethanol.

The pellet was air dried and dissolved in 500 $\mu \mathrm{L}$ TE buffer. It was stored at $-20^{\circ} \mathrm{C}$.

\section{Standardization of PCR conditions}

There are number of variables in a PCR which have to be optimized to give target amplification. These parameters are:

Denaturation temperature and time.

Annealing temperature and time.

Amount of template DNA and primer to be taken.

Concentration of $\mathrm{MgCl}_{2}$ in the assay buffer.

The number of cycles to be performed.

All the SSR markers used in the study were selected based on copy number and absence of stutter band good quality and high diversity index values (Feingold el al., 2005; Milbourne et al., 2008; Kandemir el al., 2010; Kandemir et al., 2006, Rocha et al., 2010 and Sharma et al., 2014.

Detail of SSR used in this experiment was given in table 1 . 


\section{Procedure of gel electrophoresis}

Horizontal gel electrophoresis unit was used for fractionating SSR markers on agarose gel using the following procedure:

The open ends of a clean, dry plastic tray supplied with the electrophoresis were scaled with tape so as to form a mold.

The mold was set on a horizontal section of a bench.

Agarose gel (1.5\% and 2.5\%) for SSR was prepared by dissolving appropriate amount of agarose in TAE buffer. Agarose was dissolved by heating the solution at $100^{\circ} \mathrm{C}$. It is allowed to cool to room temperature.

Added EtBr to a final concentration of $(0.5$ $\mu \mathrm{L}$ of $10 \mathrm{mg} / \mathrm{mL}$ ) solution of EtBr to $100 \mathrm{~mL}$ gel mixture) and mix well.

After complete setting of the gel, the comb was removed carefully, the tape was removed and the gel was mounted in an electrophoresis tank and PCR amplified products were loaded in each gel along with molecular markers.

A pre-run of 15 minutes at 50 Volt given to the gel.

For each well, DNA loading dye and DNA samples were mixed in 1:5 ratios and loaded in the gel with a micropipette.

Electrophoresis was done at $80-90$ volt for 3 hour in TAE electrophoresis buffer.

The gel was visualized in U.V. transilluminator (BioRad, USA) and stored using gel documentation system.

\section{Data analysis}

Amplified SSR profile of all the genotypes with each primer was documented using gel documentation stem. Data were recorded as presence (1) or absence (0) of band throughout the amplified profile with a primer. Similarly data were also recorded for all the primers separately as presence or absence of band. Each amplified band was considered as unique locus. The binary data were used to calculate genetic similarities based on Jaccard's coefficient (Jaccard, 1981) and UPGMA (Unweighted Pair Group Method using Arithmetical Averages) dendrogram was generated to determine the genetic relationship of potato genotypes.

\section{Results and Discussion}

\section{Genetic diversity analysis}

Pair wise similarity matrix based on Jaccard's coefficient for fifteen SSR primers are presented in table 2 for 50 potato genotypes. Four primers were found monomorphic and rests were found to be polymorphic. The similarity coefficient was ranged from 31 to 100 per cent. Maximum similarity occurred between EM-5 and TPSK-05-06-44 (100\%) while TPSK-05-06-95 and Laddy Rosseta also showed 100 per cent similarity with each other (Table 3).

\section{Cluster analysis}

The phylogenetic tree was constructed through NTSYSpc cluster analysis software using UPGMA (un-weighted pair group method with arithmetic mean) of SSR markers involving data generated out of fifteen primers on fifty genotypes of potato depicted in figure 1. All 50 genotypes were demarcated at approximately 51 per cent similarity and further divided into two clusters each with approximately 57.5 and 58.5 per cent similarity (Fig.1).

Cluster I comprised of 30 genotypes while cluster II contained Only 20 genotypes. 
Table.1 Detailed description of primer sequences of SSR markers for potato

\begin{tabular}{|c|c|c|c|}
\hline S. No. & SSR Primer Code & Forward Sequence (5'3') & Reverse Sequence (5ُ2’') \\
\hline 1. & STM0007 & 5'-GACAAGCTGTGAAGTTTAT-3' & 5'AATTGAGAAAGAGTGTGTGTG-3' \\
\hline 2. & STI24 & 5'-CGCCATTCTCTCAGATCACTC-3' & 5'-GCTGCAGCAGTTGTTGTTGT-3' \\
\hline 3. & STI30 & 5'-TTGACCCTCCAACTATAGATTCTTC-3' & 5'-TGACAACTTTAAAGCATATGTCAGC-3' \\
\hline 4. & STI57 & 5'-CCTTGTAGAACAGCAGTGGTC-3' & 5'-TCCGCCAAGACTGATGCA-3' \\
\hline 5. & STP0Ac58 & 5'TTGATGAAAGGAATGCAGCTTGIG-3' & 5'ACGTTAAAGAAGTGAGAGTACGAC-3' \\
\hline 6. & STM1016 & 5'TTCTGATTTCAGTCATGTTTCC-3' & 5'ATGCTTGCCATGTGATGTGT-3' \\
\hline 7. & STI031 & 5’AGGCGCACTTTAACTTCCAC-3' & 5'CGGAACAAATTGCTCTGATG-3' \\
\hline 8. & STM1104 & 5'TGATTCTCTTGCCTACTGTAATCG-3' & 5'CAAAGTGGTGTGAAGCTGTGA-3' \\
\hline 9. & STM1106 & 5'TCCAGCTGATTGGTTAGGTTG-3' & 5'ATGCCGAATCTACTCGTCATGG-3' \\
\hline 10. & STM0037 & 5'AATTTAACTTAGAAGATTAGTCTC-3' & 5’ATTTGGTTGGGTATGATA-3' \\
\hline 11. & STM0030 & 5'AGAGATCGATGAAAAACACGT-3' & 5'GTGGCATTTTGATGGATT-3' \\
\hline 12. & STM0019 & 5'AATAGGTGTACTGACTCTCAATG-3' & 5'TTGAAGTAAAAGTCCTAGTATGTG-3' \\
\hline 13. & STM1049 & 5'CTACCAGTTTGTTGATTGTGGTG-3' & 5’AGGGACTTTAATTTGTTGGACG-3' \\
\hline 14. & STM1031 & 5'TGTGTTTGTTTTTCTGTAT-3' & 5’TTCAGTCAACTCCTGTTGCG-3' \\
\hline 15. & STU6SNRN & 5'GAAGTTTTATCAGAATCC-3' & 5'ATCACCTCATCAGCAATC-3' \\
\hline 16. & STI005 & 5'CTACCAGTTTGTTGATTGTGGTG-3' & 5'AGGGACTTTAATTTGTTGGACG-3' \\
\hline 17. & STGBSS & 5'AATCGGTGATAAATGTGAATGC-3' & 5’ATGCTTGCCATGTGATGTGT-3' \\
\hline 18. & STWAX-2 & 5'CCCATAATACTGTCGATGAGCA-3' & 5'GAATGTAGGGAAAGATGCATGA-3' \\
\hline 19. & STCAAS3 & 5'AATTCATGTTTGCGGTAGGTC-3' & 5'ATGCAGAAAGATGTCAAAATTGA-3' \\
\hline
\end{tabular}

Table.2 Analysis for polymorphism in SSR markers

\begin{tabular}{|c|c|c|c|c|c|c|c|c|}
\hline $\begin{array}{l}\text { S. } \\
\text { No. }\end{array}$ & Primer & $\begin{array}{c}\text { Sample } \\
\text { size }\end{array}$ & $\begin{array}{c}\text { GC } \\
\text { content }(F)\end{array}$ & $\begin{array}{c}\text { GC } \\
\text { content }(R)\end{array}$ & $\begin{array}{c}\text { Annealing } \\
\text { temperature }\left({ }^{\circ} \mathrm{C}\right)\end{array}$ & $\begin{array}{c}\text { Polymorphic } \\
\text { bands }\end{array}$ & $\begin{array}{c}\text { Monomorphic } \\
\text { bands }\end{array}$ & $\begin{array}{c}\text { Polymorphism } \\
(\%)\end{array}$ \\
\hline 1. & STM0007 & 48.00 & 36.84 & 38.09 & 58.00 & 3 & 0 & 100 \\
\hline 2. & STI24 & 48.00 & 52.38 & 50.00 & 60.00 & 4 & 0 & 100 \\
\hline 3. & STI57 & 48.00 & 52.38 & 55.55 & 60.00 & 3 & 1 & 75 \\
\hline 4. & STP0Ac58 & 48.00 & 41.66 & 41.66 & 57.00 & 2 & 0 & 100 \\
\hline 5. & STM1016 & 48.00 & 31.81 & 45.00 & 58.00 & 2 & 0 & 100 \\
\hline 6. & STM0037 & 48.00 & 25.00 & 33.33 & 53.00 & 2 & 0 & 100 \\
\hline 7. & STM0030 & 48.00 & 33.33 & 38.55 & 55.00 & 2 & 0 & 100 \\
\hline 8. & STM0019 & 48.00 & 39.13 & 33.33 & 52.00 & 2 & 0 & 100 \\
\hline 9. & STI005 & 48.00 & 39.13 & 40.90 & 58.50 & 5 & 0 & 100 \\
\hline 10. & STGBSS & 48.00 & 36.36 & 45.00 & 58.80 & 6 & 0 & 100 \\
\hline 11. & STM1031 & 48.00 & 26.31 & 50.00 & 52.60 & 3 & 1 & 75 \\
\hline
\end{tabular}


Table.3 Principal component (PC) score based on the correlation coefficient matrix of 17 quantitative characters of

50 genotypes of potato (2014-15)

\begin{tabular}{|c|c|c|c|c|c|c|c|}
\hline Genotype & Vector 1 & Vector 2 & Vector 3 & Vector 4 & Vector 5 & Vector 6 & Vector 7 \\
\hline Kufri Arun & 338.441 & 51.838 & 132.575 & -197.283 & -113.521 & 108.314 & 107.950 \\
\hline Kufri Jawahar & 250.191 & 9.631 & 117.667 & -147.301 & -83.968 & 70.225 & 70.526 \\
\hline PNT-1 & 252.036 & 6.552 & 112.538 & -145.410 & -86.139 & 59.336 & 63.608 \\
\hline TPSK-05-06-110 & 155.756 & -48.683 & 110.550 & -98.414 & -53.167 & 21.888 & 19.748 \\
\hline TPSK-05-06-79 & 291.725 & 1.142 & 134.252 & -174.679 & -102.380 & 76.383 & 72.666 \\
\hline MS/95-1542 & 277.499 & 17.028 & 110.840 & -158.730 & -106.532 & 45.449 & 65.920 \\
\hline EM-2 & 227.724 & -0.596 & 116.541 & -135.529 & -81.265 & 51.261 & 56.007 \\
\hline TPSK-0506-98 & 186.515 & -42.734 & 122.380 & -113.317 & -65.692 & 36.003 & 30.390 \\
\hline DPS-19 & 185.722 & -18.471 & 112.542 & -106.607 & -60.340 & 40.466 & 39.130 \\
\hline M-3 & 276.990 & 26.624 & 128.865 & -164.292 & -97.837 & 80.061 & 82.679 \\
\hline TPSK-05-06-95 & 84.663 & -89.899 & 106.939 & -63.583 & -37.529 & -10.777 & -16.108 \\
\hline Laddy Rossetta & 121.689 & -66.799 & 117.273 & -79.879 & -44.396 & 12.137 & 7.733 \\
\hline EM-5 & 180.412 & -24.237 & 112.302 & -114.306 & -63.747 & 35.121 & 35.298 \\
\hline EM-3 & 186.060 & -21.699 & 118.425 & -118.604 & -65.854 & 41.333 & 38.082 \\
\hline Kufri Giriraj & 304.545 & 17.640 & 138.041 & -182.105 & -115.488 & 77.774 & 81.795 \\
\hline Atlanta & 109.602 & -64.765 & 110.051 & -63.766 & -39.632 & 4.686 & -0.358 \\
\hline Kufri Sadabahar & 82.454 & -81.710 & 100.798 & -57.768 & -31.496 & -9.595 & -13.734 \\
\hline AICRP-07-05 & 205.315 & -41.087 & 121.602 & -125.231 & -83.417 & 25.373 & 26.630 \\
\hline TPSK-05-06-117 & 328.164 & -5.004 & 144.379 & -205.661 & -137.598 & 65.098 & 70.130 \\
\hline Kufri Chipsona-2 & 177.313 & -50.884 & 124.969 & -104.974 & -72.515 & 14.518 & 16.746 \\
\hline MS/93-1344 & 143.307 & -66.670 & 114.979 & -97.428 & -57.504 & 10.826 & 6.715 \\
\hline TPSK-05-06-105 & 146.721 & -54.289 & 108.916 & -89.967 & -61.197 & 9.411 & 12.132 \\
\hline Kufri Badsah & 160.979 & -30.624 & 113.258 & -91.297 & -53.574 & 34.167 & 29.774 \\
\hline TPSK-05-06-80 & 151.279 & -57.551 & 111.950 & -93.155 & -55.525 & 14.606 & 12.828 \\
\hline Kufri Pushkar & 303.811 & 11.610 & 128.645 & -183.012 & -114.406 & 74.516 & 74.100 \\
\hline TPSK -05-06-44 & 290.809 & -30.134 & 121.613 & -174.713 & -128.074 & 28.219 & 44.189 \\
\hline Kufri Khyati & 225.743 & -22.409 & 122.915 & -129.453 & -84.043 & 42.688 & 39.936 \\
\hline Kufri Jyoti & 322.296 & 35.848 & 131.710 & -188.128 & -118.069 & 89.900 & 95.167 \\
\hline TPSK-05-06-61 & 136.818 & -67.465 & 108.352 & -81.825 & -53.788 & 0.398 & 2.523 \\
\hline EM-1 & 162.553 & -22.989 & 109.276 & -98.506 & -49.827 & 41.399 & 35.075 \\
\hline TPSK-05-06-85 & 191.139 & -54.996 & 123.202 & -115.576 & -73.715 & 20.626 & 17.904 \\
\hline $\mathrm{C}-11$ & 157.175 & -29.005 & 115.566 & -90.143 & -49.709 & 36.870 & 33.381 \\
\hline DPS-07 & 250.286 & 14.034 & 117.733 & -151.936 & -83.459 & 70.777 & 71.370 \\
\hline TPSK-05-06-86 & 162.542 & -55.807 & 117.781 & -98.920 & -61.611 & 14.155 & 12.921 \\
\hline MS/99-1871 & 154.579 & -61.727 & 113.781 & -108.766 & -61.023 & 13.438 & 12.362 \\
\hline $\mathrm{MS} / 0-3740$ & 305.907 & 2.921 & 134.991 & -198.673 & -113.497 & 78.864 & 77.162 \\
\hline TPSK-05-06-83 & 349.377 & 10.829 & 145.151 & -221.950 & -135.450 & 83.118 & 86.552 \\
\hline TPSK-05-06-007 & 264.759 & -15.001 & 135.904 & -162.829 & -99.677 & 56.086 & 56.022 \\
\hline Kufri Himalini & 257.414 & 19.267 & 117.702 & -149.780 & -85.868 & 71.900 & 73.858 \\
\hline
\end{tabular}




\section{Int.J.Curr.Microbiol.App.Sci (2017) 6(7): 4219-4230}

\begin{tabular}{|c|c|c|c|c|c|c|c|}
\hline Kufri Surya & 263.158 & 29.143 & 126.245 & -150.200 & -81.267 & 84.695 & 80.317 \\
\hline MS/0-9808 & 203.099 & -12.524 & 114.619 & -120.664 & -68.993 & 44.887 & 44.122 \\
\hline PH-3 & 199.416 & -34.361 & 123.466 & -116.757 & -76.833 & 30.265 & 31.567 \\
\hline $\mathrm{P}-11$ & 186.919 & -27.863 & 111.185 & -111.504 & -72.651 & 30.672 & 31.465 \\
\hline $\mathrm{C}-1$ & 133.516 & -30.120 & 85.506 & -73.983 & -48.510 & 8.890 & 19.613 \\
\hline $\mathrm{C}-10$ & 199.515 & -2.025 & 105.046 & -113.916 & -70.454 & 39.144 & 47.538 \\
\hline Kufri Frysona (C) & 272.355 & -1.542 & 129.661 & -168.878 & -106.508 & 57.120 & 62.851 \\
\hline Kufri Chipsona-1 (C) & 287.018 & 26.895 & 121.061 & -165.395 & -98.977 & 72.998 & 79.969 \\
\hline Kufri Bahar (C) & 96.165 & -62.732 & 97.608 & -66.726 & -33.249 & 0.423 & -1.504 \\
\hline Kufri Guarav (C) & 251.535 & -14.709 & 118.747 & -148.158 & -100.129 & 40.041 & 50.464 \\
\hline Kufri Ashoka (C) & 307.667 & 18.192 & 138.361 & -185.396 & -109.823 & 88.379 & 80.407 \\
\hline
\end{tabular}

Fig.1 Dendogram depicted the classification of the fifty genotypes of potato constructed using UPGMA method based on

SSR markers. The scale in the bottom is Jaccard's coefficient of similarity
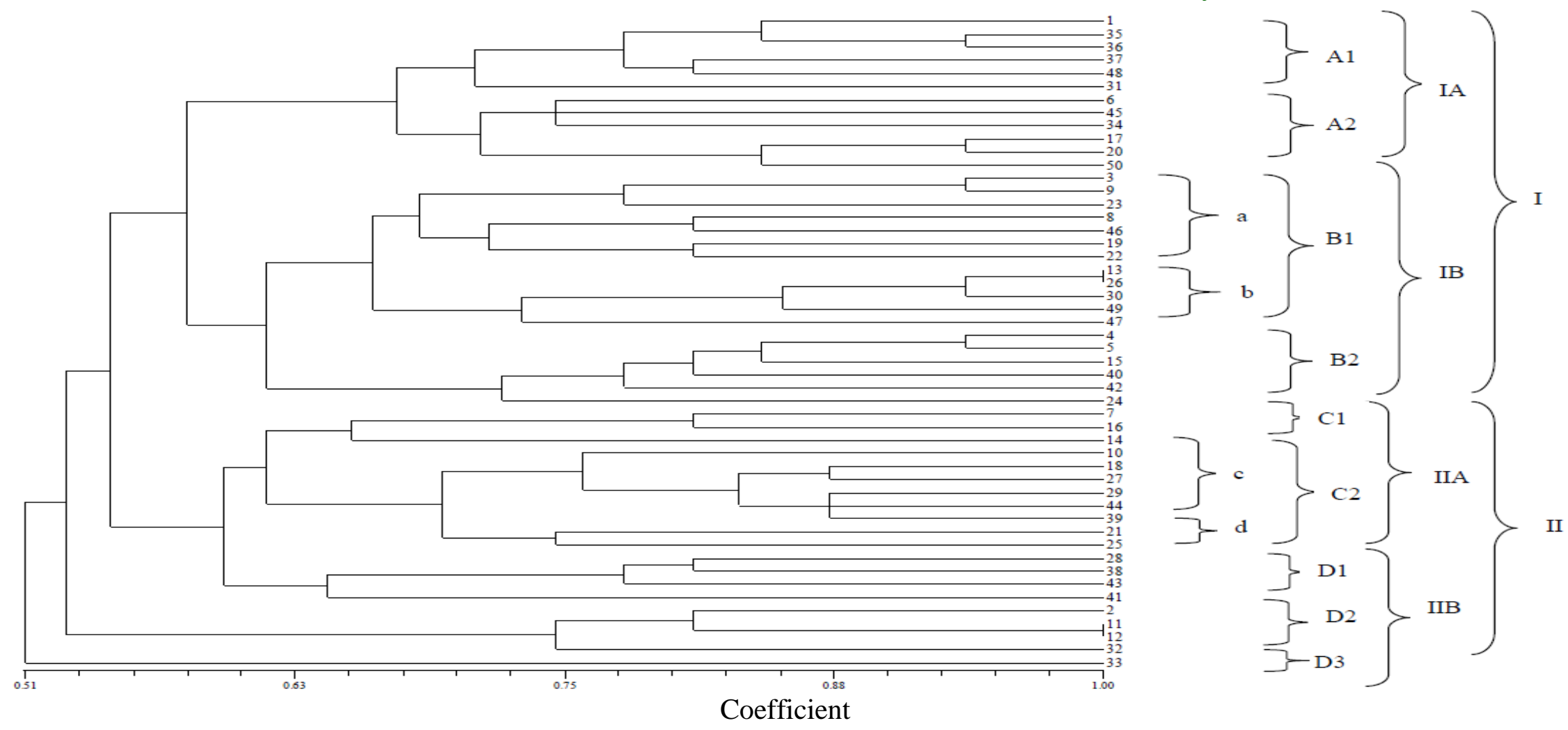
Fig.2 PCR amplification of 50 genotypes of potato by SSR primer STI24

$\begin{array}{lllllllllllllllllllllllll}1 & 2 & 3 & 4 & 5 & 6 & 7 & 8 & 9 & 10 & 11 & 12 & 13 & 14 & 15 & 16 & 17 & 18 & 19 & 20 & 21 & 22 & 23 & 24 & 25\end{array}$

1000

500

300

200

100

1000

500

300

200

100

Fig.3 PCR amplification of 50 genotypes of potato by SSR primer STI57

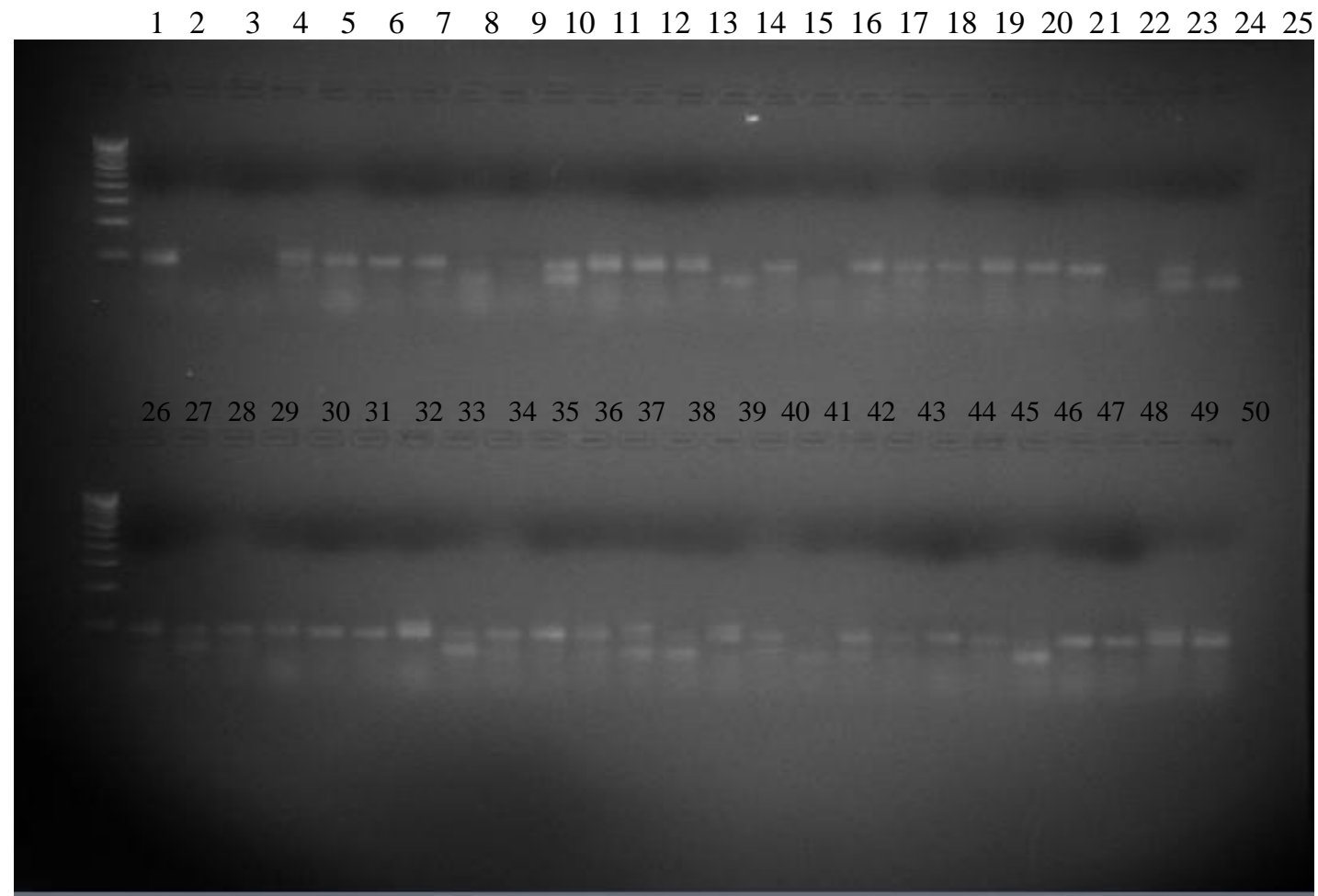

1000

500

300

200

100

1000

500

300

200

100

$\begin{array}{lllllllllllllllllllllllll}1 & 2 & 3 & 4 & 5 & 6 & 7 & 8 & 9 & 10 & 11 & 12 & 13 & 14 & 15 & 16 & 17 & 18 & 19 & 20 & 21 & 22 & 23 & 24 & 25\end{array}$

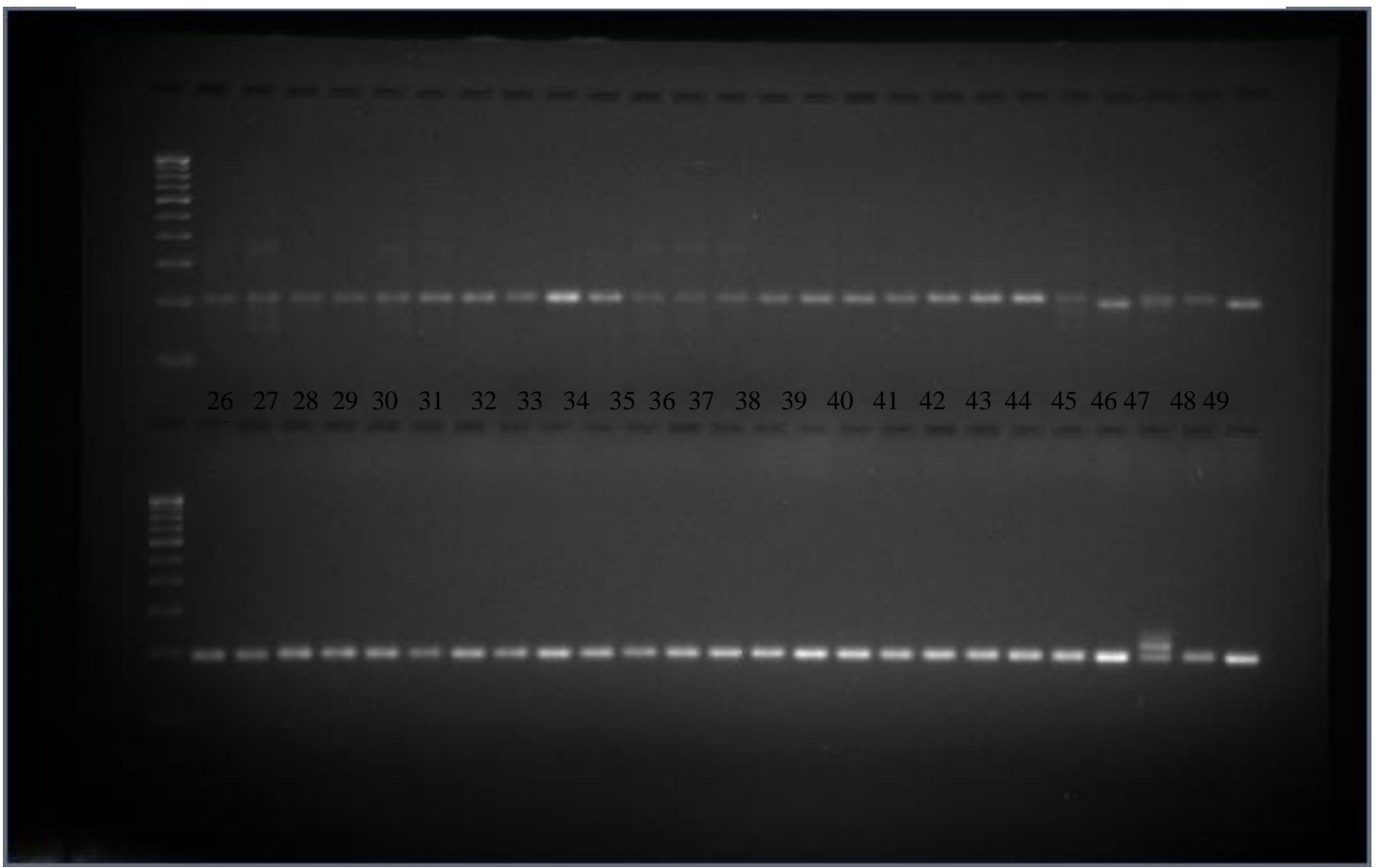


Fig.4 PCR amplification of 50 genotypes of potato by SSR primer STGBSS

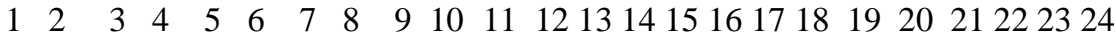

1000

500

300

200

100

$26 \quad 2728 \quad 2930 \quad 3132 \quad 33 \quad 34 \quad 35 \quad 36 \quad 37 \quad 38 \quad 39 \quad 40 \quad 41 \quad 42 \quad 43 \quad 444546 \quad 47 \quad 48 \quad 49 \quad 50$

1000

500

300

200

100

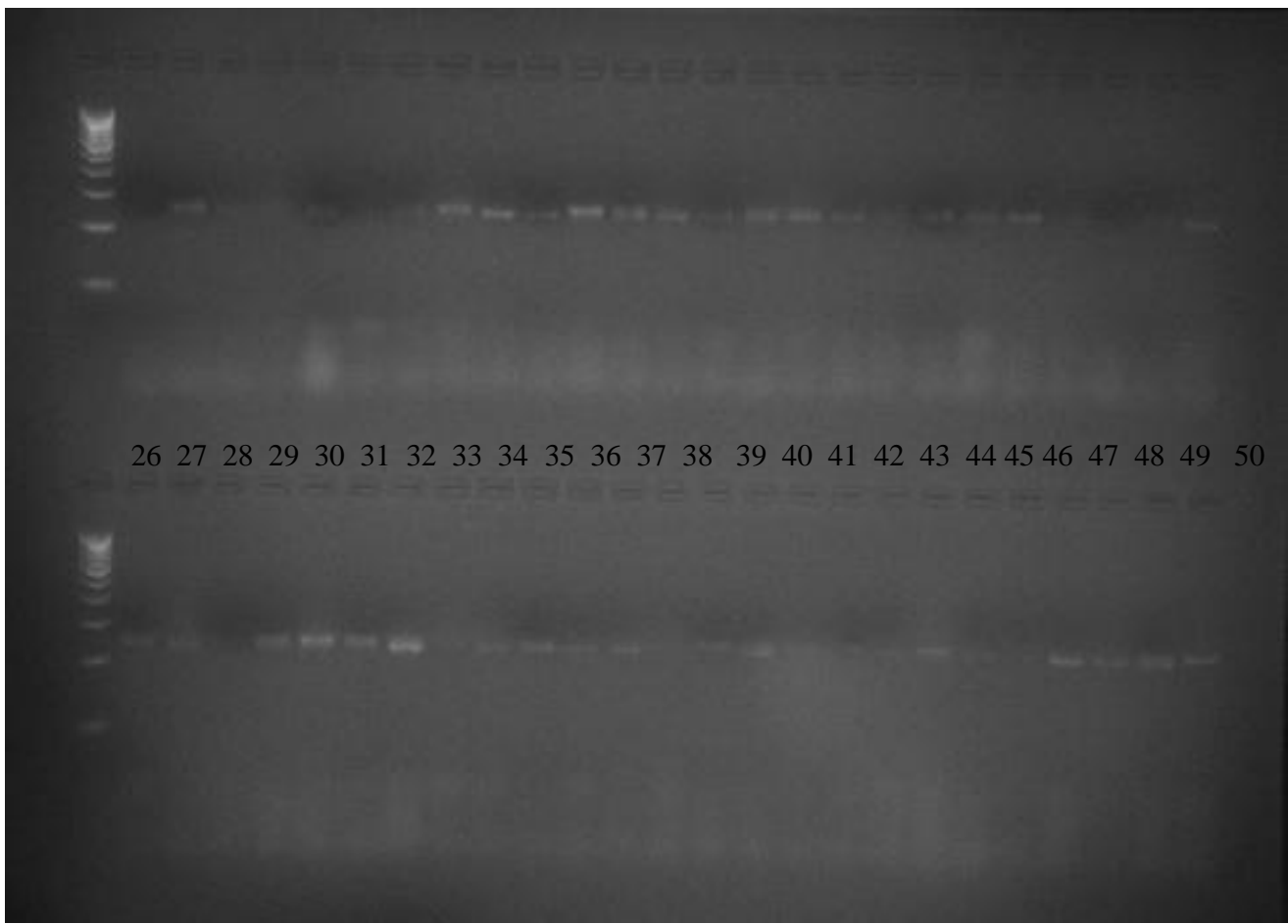

Fig.5 PCR amplification of 50 genotypes of potato by SSR primer STM1016

$\begin{array}{lllllllllllllllllllllllll}1 & 2 & 3 & 4 & 5 & 6 & 7 & 8 & 9 & 10 & 11 & 12 & 13 & 14 & 15 & 16 & 17 & 18 & 19 & 20 & 21 & 22 & 23 & 24 & 25\end{array}$

1000

500

300

200

100

1000

500

300

200

100

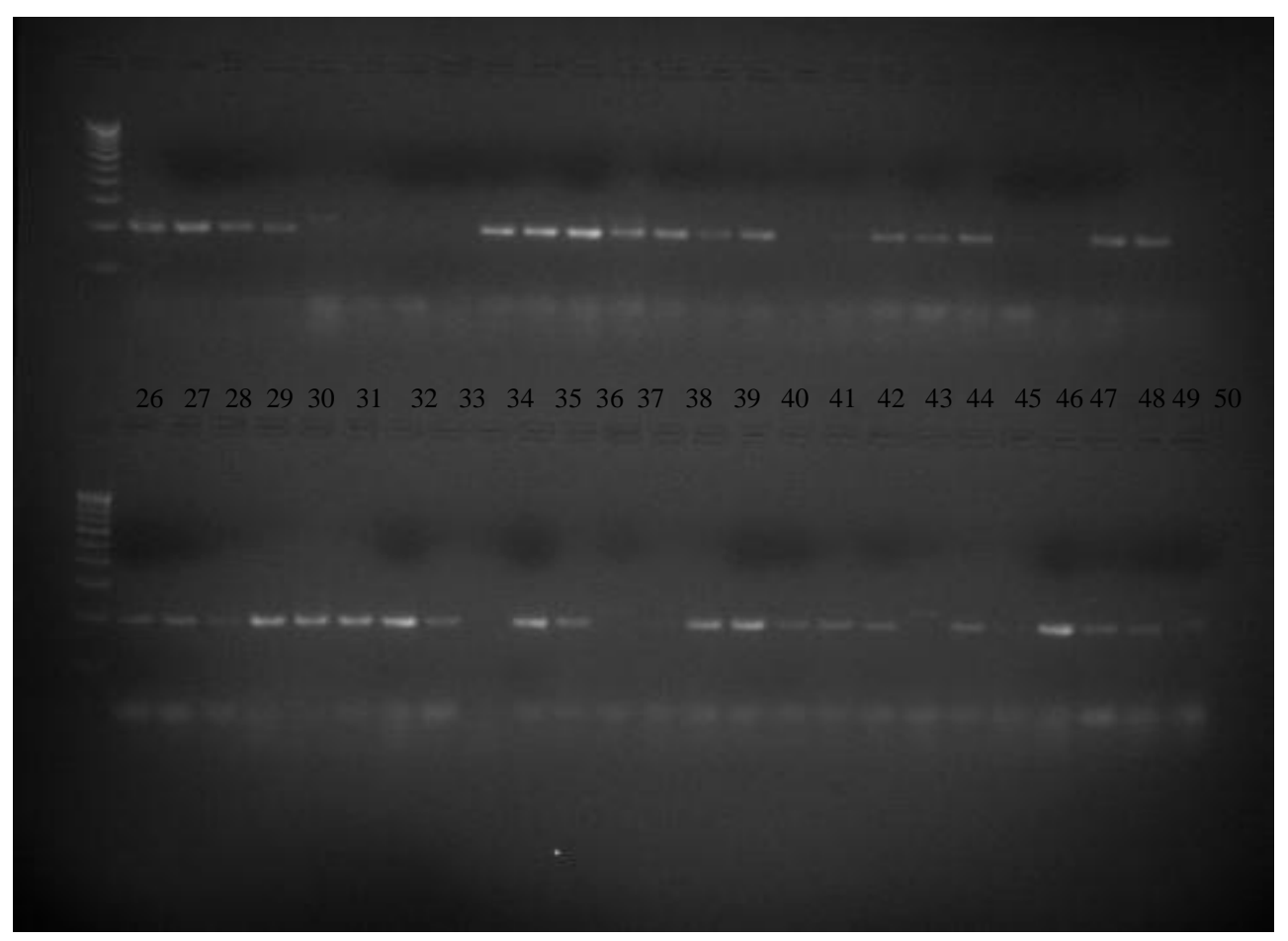


Int.J.Curr.Microbiol.App.Sci (2017) 6(7): 4219-4230

Fig.6 PCR amplification of 50 genotypes of potato by SSR primer STP0Ac58

$\begin{array}{lllllllllllllllllllllllll}1 & 2 & 3 & 4 & 5 & 6 & 7 & 8 & 9 & 10 & 11 & 12 & 13 & 14 & 15 & 16 & 17 & 18 & 19 & 20 & 21 & 22 & 23 & 24 & 25\end{array}$

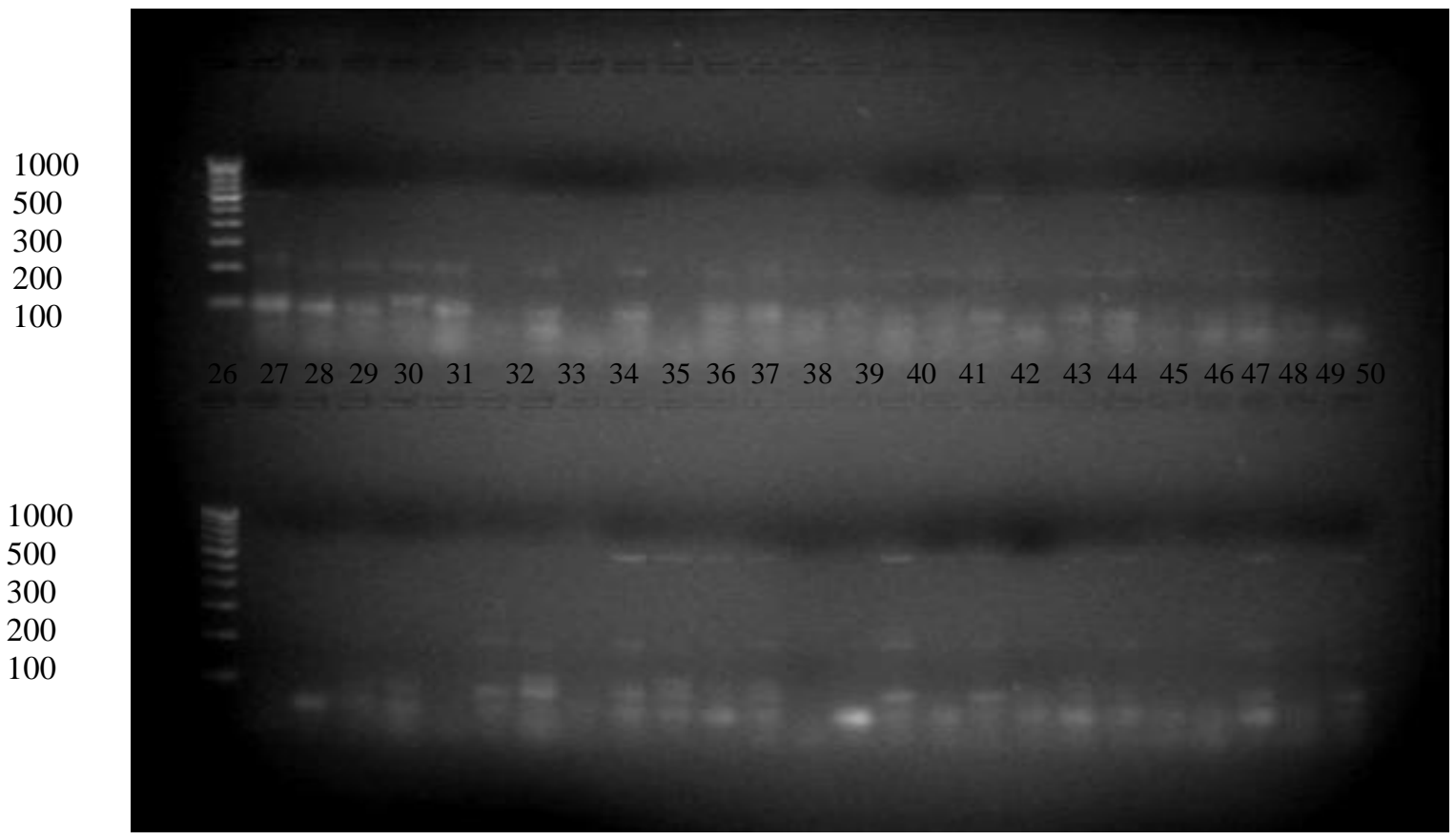

Fig.7 PCR amplification of 50 genotypes of potato by SSR primer STM0019

$\begin{array}{lllllllllllllllllllllllll}1 & 2 & 3 & 4 & 5 & 6 & 7 & 8 & 9 & 10 & 11 & 12 & 13 & 14 & 15 & 16 & 17 & 18 & 19 & 20 & 21 & 22 & 23 & 24\end{array}$

1000

500

300

200

100

1000

500

300

200

100

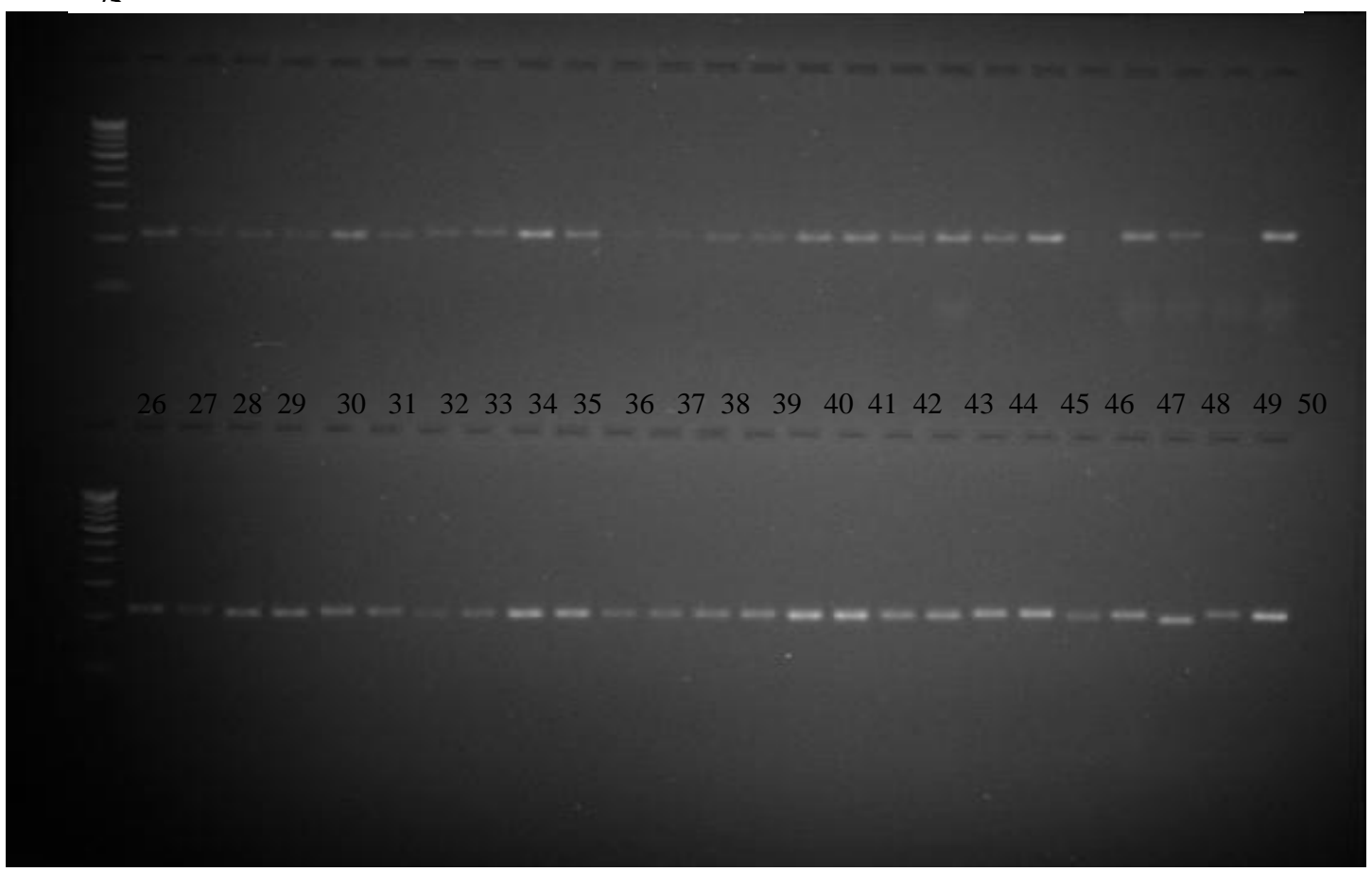


Fig.8 PCR amplification of 50 genotypes of potato by SSR primer STI005

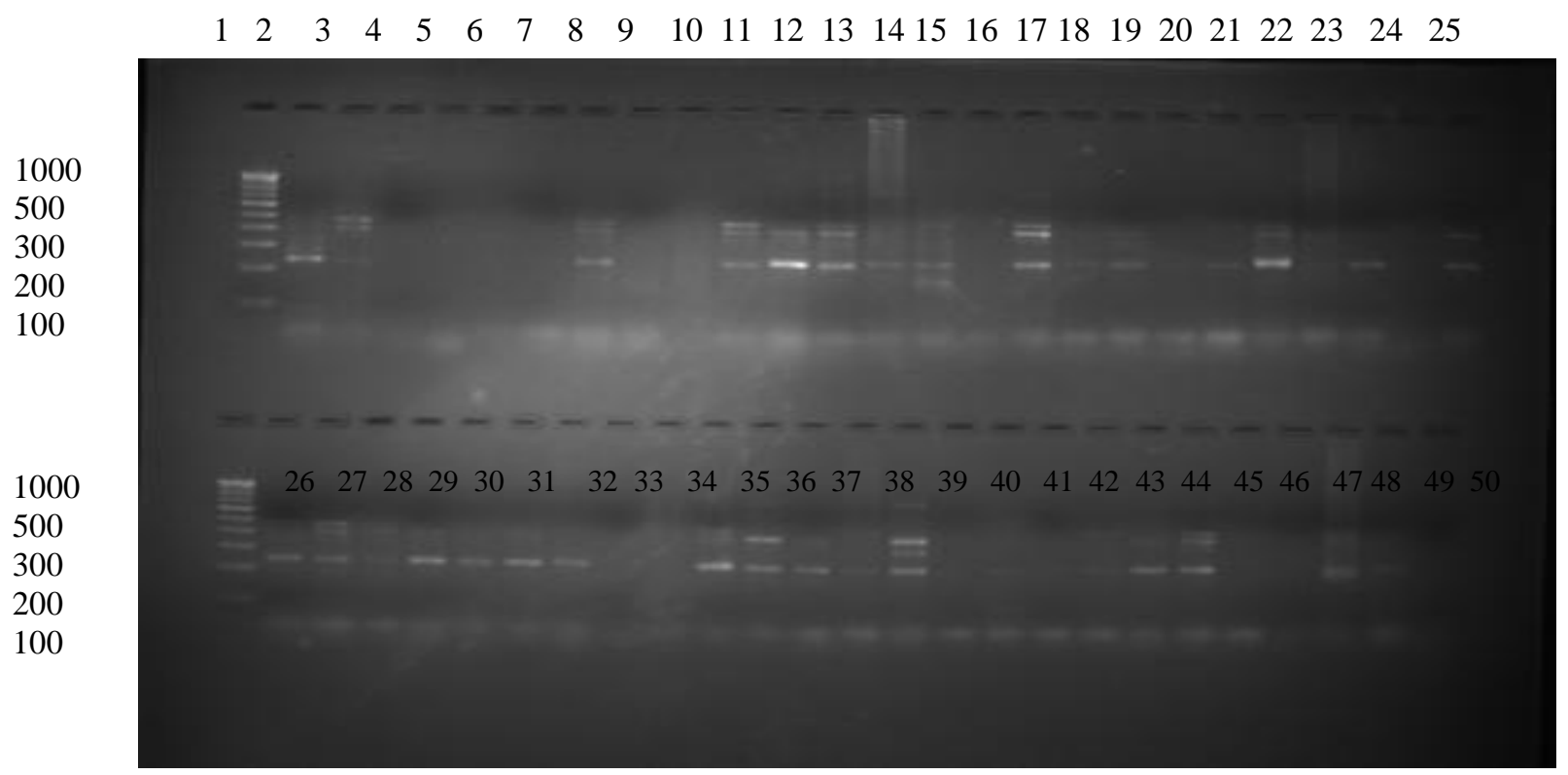

Fig.9 PCR amplification of 50 genotypes of potato by SSR primer STM0030
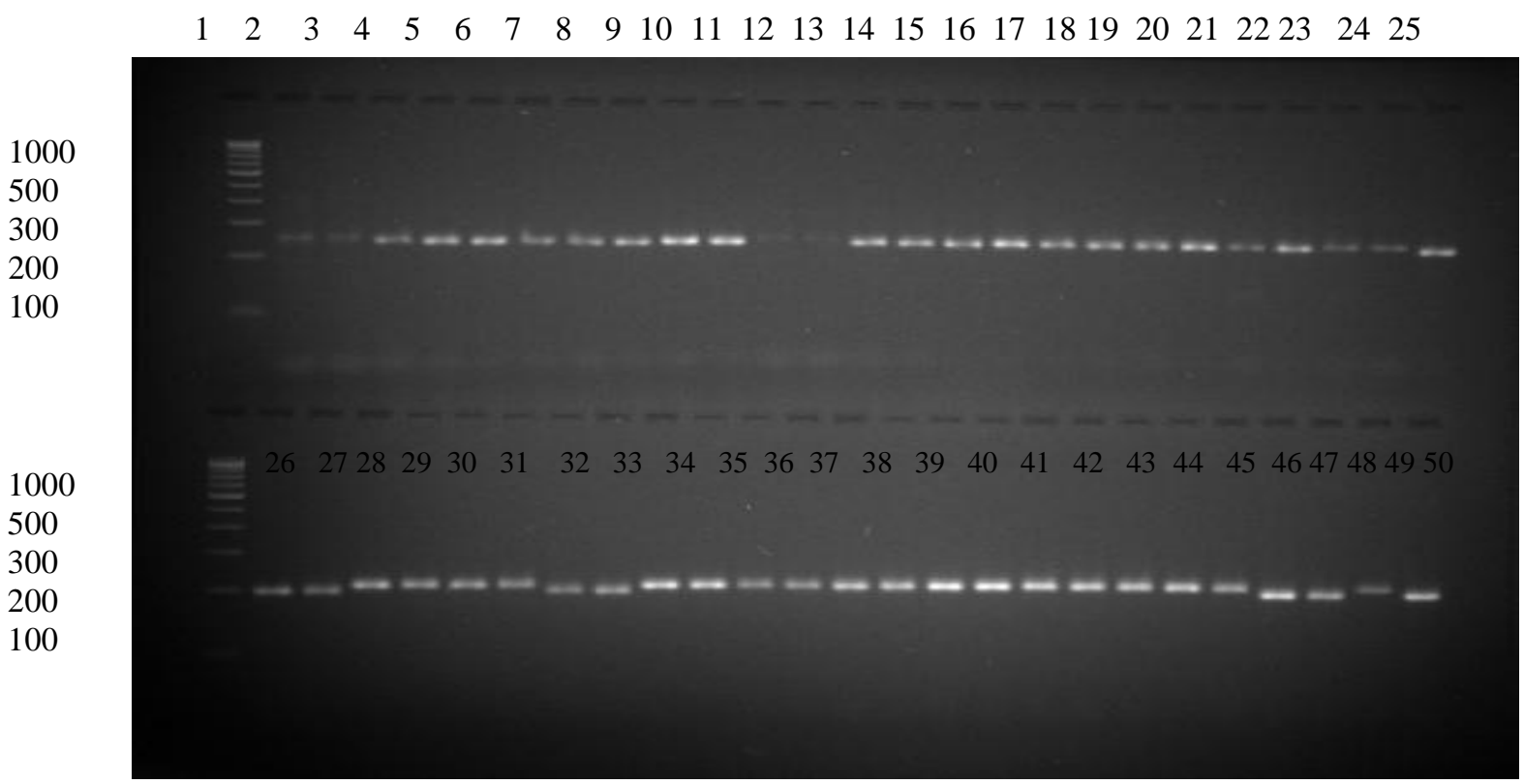

Cluster I was further subdivided into two subclusters IA and IB each with approximately 67 and 61.5 per cent similarity, respectively. Subcluster IA was further forked in to two small clusters $\mathrm{Al}$ and $\mathrm{A} 2$, cluster $\mathrm{Al}$ genotypes had approximately 70 per cent similarity and contain 6 genotypes i.e. Kufri Arun, MS/991871, MS/0-3740, TPSK-05-06-83, Kufri Bahar and TPSK-05-06-85 while cluster A2 also contain 6 genotypes (MS/95-1542, C-10, TPSK-05-06-86, Kufri Sadabahar, Kufri Chipsona-2, Kufri Ashoka) with approximately 71 per cent similarity. Sub cluster IB divided into two small clusters B1 and B2 with approximately 66 and 72 per cent similarity. Cluster Bl again divided into two super small cluster $\mathrm{a}$ and $\mathrm{b}$ with approximately 68 and 74 per cent similarity. Cluster a of super small 
cluster comprised seven genotypes viz. PMT-1, DPS-19, Kufri Badsah, TPSK-05-06-98, Kufri Frysona, TPSK-05-06-117 and TPSK-05-06105. Cluster b of super small clusters contains five genotypes EM-5, TPSK-05-06-44, EM-1, Kufri Gaurav and Kufri Chipsona-1. Cluster B2 contain six genotypes i.e. TPSK-05-06-110, TPSK-05-06-79, Kufri Giriraj, Kufri Surya, PH3 and TPSK-05-06-80.

Main cluster II was further subdivided into two sub-clusters IIA and IIB each with approximately 61.5 and 64 per cent similarity, respectively.

Sub-cluster IIA was further forked in to two small clusters $\mathrm{C} 1$ and $\mathrm{C} 2$, cluster $\mathrm{C} 1$ genotypes had approximately 64.5 per cent similarity and contain 3 genotypes i.e. EM-2, Atlanta and EM3 while cluster $\mathrm{C} 2$ further divided into super small cluster i.e. c and d. Super small cluster c contain six genotypes viz.M-3, AICRP-07-05, Kufri Khyati, TPSK-05-06-61, C-1 and TPSK05-06-007 with 75.5 per cent similarity and cluster d of super small cluster comprised two genotypes viz. MS/93-1344 and Kufri Pushkar with 74.8 per cent similarity. Cluster IIB divided into two small cluster i.e. cluster D1, cluster D2 and cluster D3 with approximately 64, 74.5 and 51 per cent similarity. Cluster D1 conatin Kufri Jyoti, TPSK-05-06-007, P-11 and MS/0-9808. Cluster D2 contain four genotypes i.e. Kufri Jawahar, TPSK-05-06-95, Laddy Rossetta and C-11. Cluster D3 comprised one genotype i.e. DPS-07.

In contrast, SSRs are codominant and give rereproducible results because they are mostly developed from introns of genes that is why they are said to be highly specific especially useful for mapping in tetraploid potato. Milbourne et al., 1997 compared different types of PCR derived markers to estimate variability and concluded that microsatallites offer an effective means of analyzing genetic distance between potato varieties. SSRs additional advantages include their ubiquity, distribution across the genome, co-dominant behavior, multiallelism, reproducibility and high level of polymorphism detected (Milbourne et al., 1997, Mc Gregor et al., 2000 and Spooner et al., 2005). SSRs produced specific patterns, high polymorphism and placed genotypes in many clusters. Similar findings were recorded by Ghislain et al., (2006) and Komy et al., (2012). Thus, by means of SSR markers the genetic diversity was assessed and the 50 potato genotypes analyzed in this study were identified (Figs. 2-9).

Thus, based on the finding of present investigation it can be concluded that significant diversity and variability was present among the genotypes. The SSR markers used in the study were found to produce polymorphic bands and resulted into differentiation among various genotypes under study. SSRs produced specific patterns, high polymorphism and placed genotypes in clusters. Thus, by means of SSR markers the genetic diversity assessed and the 50 potato genotypes/varieties analyzed in this study were identified. The significant variation exists among the genotypes based on molecular characters and with the use of SSR markers, assessment of the genetic diversity can also help us to plan a future breeding programme using the diverse parent.

\section{References}

Braun, A and Wenzel, Z. 2005. Molecular analysis in genetic variation in potato (Solanum tuberosum L.). german cultivars and advanced clones. Potato Res., 47:8192.

Chakrabarti, S. K.; Pattanayak, D. and Naik, P. S. 2001. Figerprinting Indian potato cultivars by Random Amplified Polymorphic DNA (RAPD) markers. Potato Res., 44:375-387.

Feingold, S.; Lloyd, J.; Norero, N.; Bonierbale, M. and Lorenzen, J. 2005. Mapping and characterization of new EST derived microsatellite for potato (Solanum tuberosum L.). Theor Appl Genet., 111:456-466.

Gaur, P.C.; Kishore, H. and Gupta, P.K. 1978. 
Studies on genetic divergence in potato. Euphytica, 27: 361-368.

Ghislain, M.; Spooner, D.M.; Rodriguez, F.; Villamon, F.; Nun ez, J.; Va' squez, C.; Waugh, R and, Bonierbale, M. 2006. Selection of highly informative and userfriendly microsatellites (SSRs) for genotyping of cultivated potato. Theor. Appl. Genet., 108: 881-890.

Hayder, A. M.B.; Ahmed, M.M.; Hannan, M.A.; Razvy, M.A.; Mandal, M.; Salahin, R.; Karim and Hossain, K. M., 2007. Analysis of genetic diversity in some potato varieties grown in Bangladesh. Middle-East J. Scientific Res., 2(3-4): 143-145.

Kandemir, N.; Yilmaz, G.; Karan, Y. B. and Borazan, D. 2006. Development of a Simple Sequence repeat (SSR) marker set to fingerprint local and modern potato varieties grown in central Anatolian Plateau in Turkey. Afr. J. Biotechnol., 9(34): 55165522.

Kandemir, N.; Yilmaz, G.; Karan, Y. B. and Borazan, D. 2010b. Isolation of different genotypes in Basciftlik beyazi potato landrace using SSR markers. Turkish Journal of Field Crops, 15(1): 84-88.

Komy, M.H.; Saleh, A.A. and Molan, Y.Y. 2012. Molecular characterization of early blight disease resistant and susceptible potato cultivars using RAPD and SSR markers. African J. of Biotech, 11(1): 3745.

McGregor, C.E.; Lambert, M.M.; Greyling, J.H. and Warnich, L. 2000. A comparative assessment of DNA fingerprinting technique (RAPD, ISSR, AFLP and SSR) in tetraploid potato (Solanum tuberosum L.) germplasm. Euphytica, 113:135-144.

Milbourne,, D.; Meyer, R.C.; Bradshaw, J. E.;
Baird, E.; Bonar, N.; Provan, J.; Powell. W. and Waugh, R. 1997. Comparison of PCR-based marker systems for the analysis of genetic relationship in cultivated potato. Mol. Breeding, 3: 127136.

Milbourne,, D.; Meyer, R.C.; Bradshaw, J. E.; Baird, E.; Bonar, N.; Provan, J.; Powell. W. and Waugh, R. 2008. Comparison of PCR-based marker systems for the analysis of genetic relationship in cultivated potato. Mol. Breeding, 3: 127136.

Rauf, S.; Silva, J.A.T.; Khan, A. A. and Naveed, A. 2010. Consequences of plant breeding on genetic diversity. International J. Plant Bred, 4(1):1-21.

Rocha, E. A.; Paiva, L. V.; de Carvalho, H. H. and Guimaraes, C. T. 2010. Molecular characterization and genetic diversity of potato cultivars using SSR and RAPD markers. Crop Breed. And Applied Biotechnol, 10:204-210.

Sharma, V. and Nandineni, M.R. 2014. Assessment of genetic diversity among Indian potato (Solanum tuberosum L.) collection using microsatellite and retrotransposon based marker systems. Molecular Phylogenetics and Evolution, 73: 10-17.

Shekhawat, G.S. 2001. Potato production, utilization and marketing in India. $J$. Indian PotatoAssoc, 28 (2-4): 34-36.

Spooner, D.M.; Nunez, J.; Trujillo, G.; Herrera, M.R.; Guzman, F. and Ghislain, M. 2005. Extensive simple sequence repeats genotyping of potato landraces supports a major reevaluation of their gene pool structure and classification. Proc. Natl. Acad. Sci., 104:19398-19403.

\section{How to cite this article:}

Rajani, Dhirendra Singh and Jeena, A.S. 2017. Assessing Genetic Diversity of Potato (Solanum tuberosum) Genotypes Grown in Tarai Region of Uttarakhand by Using Simple Sequence Repeat (SSR) Technique. Int.J.Curr.Microbiol.App.Sci. 6(7): 4219-4230.

doi: https://doi.org/10.20546/ijcmas.2017.607.437 